\title{
A CONTINUITY CHARACTERIZATION OF ASPLUND SPACES
}

\author{
J. R. GILES
}

(Received 5 August 2010)

\begin{abstract}
A Banach space is an Asplund space if every continuous gauge has a point where the subdifferential mapping is Hausdorff weak upper semi-continuous with weakly compact image. This contributes towards the solution of a problem posed by Godefroy, Montesinos and Zizler.
\end{abstract}

2010 Mathematics subject classification: primary 46B22; secondary 58C20.

Keywords and phrases: Fréchet differentiability, subdifferential mapping, weak upper semi-continuity, gauge, extreme point, polar, Radon-Nikodým property.

\section{Introduction}

A continuous convex function $\phi$ on a nonempty open convex subset $A$ of a Banach space $(X,\|\cdot\|)$ is said to be strongly subdifferentiable at $x \in A$ if, given $\epsilon>0$, there exists $\delta>0$ such that

$$
0 \leq \phi(x+y)-\phi(x)-\phi_{+}^{\prime}(x)(y) \leq \epsilon\|y\| \text { for all }\|y\|<\delta,
$$

and was the subject of an interesting study in [11]. The function $\phi$ is Fréchet differentiable at $x$ if also $\phi_{+}^{\prime}(x)(y)$ is linear in $y$.

A Banach space $X$ is an Asplund space if every continuous convex function $\phi$ on a nonempty open convex subset $A$ of $X$ is Fréchet differentiable at the points of a dense $G_{\delta}$ subset of $A$. It is known that $X$ is an Asplund space if $X$ possesses an equivalent strongly subdifferentiable norm; see [5, Theorem 5.1, p. 68] and [9, Proposition 8, p. 64]. Further, if $X$ is separable then it is an Asplund space if every equivalent norm has a nonzero point where it is strongly subdifferentiable [12, Theorem 1, p. 494]. It has remained an open question whether this last result can be extended to nonseparable spaces [12, Problem 6(v), p. 501]. Our aim here is to work towards such an extension.

Given a continuous convex function $\phi$ on a nonempty open convex subset $A$ of a Banach space $X$, the subdifferential of $\phi$ at $x \in A$ is the nonempty weak* compact convex subset

$$
\partial \phi(x) \equiv\left\{f \in X^{*}: f(y) \leq \phi_{+}^{\prime}(x)(y) \text { for all } y \in X\right\} .
$$

(C) 2011 Australian Mathematical Publishing Association Inc. 0004-9727/2011 \$16.00 
The set-valued subdifferential mapping $x \mapsto \partial \phi(x)$ is always Hausdorff weak ${ }^{*}$ upper semi-continuous on $A$; that is, given $x \in A$ and weak* open neighbourhood $W$ of 0 in $X^{*}$, there exists $\delta>0$ such that

$$
\partial \phi(B(x ; \delta)) \subseteq \partial \phi(x)+W
$$

by [13, Proposition 2.5, p. 19]. Further, $\phi$ is strongly subdifferentiable at $x \in A$ if and only if, given $\epsilon>0$, there exists $\delta>0$ such that

$$
\partial \phi(B(x ; \delta)) \subseteq \partial \phi(x)+\epsilon B\left(X^{*}\right)
$$

by $[8$, Theorem 3.2, p. 28]. We work with the slightly weaker property; we say that the subdifferential mapping $x \mapsto \partial \phi(x)$ is Hausdorff weak upper semi-continuous at $x \in A$ if, given a weak open neighbourhood $V$ of 0 in $X^{*}$, there exists $\delta>0$ such that

$$
\partial \phi(B(x ; \delta)) \subseteq \partial \phi(x)+V .
$$

This has been studied in [1] and for subdifferentials of the norm in [10]. Contreras and Payá showed that $X$ is an Asplund space if it possesses an equivalent norm whose subdifferential mapping is Hausdorff weak upper semi-continuous on its unit sphere [3, Theorem 1.2, p. 453]. Here we show that $X$ is an Asplund space if every continuous gauge $p$ on $X$ has a point in its domain where the subdifferential mapping $x \mapsto \partial p(x)$ is Hausdorff weak upper semi-continuous with weakly compact image. (We note that every continuous gauge $p$ on a Banach space $X$ is always strongly differentiable at 0 so is always Hausdorff weak upper semi-continuous there but the subdifferential $\partial p(0)$ is not in general weakly compact.)

\section{The density property}

We explore the effect of Hausdorff weak upper semi-continuity on higher dual spaces. Consider a bounded closed convex set $K$ with $0 \in$ int $K$ in a Banach space $X$. The gauge $p$ of $X$ defined by

$$
p(x) \equiv \inf \{\lambda>0: x \in \lambda K\}
$$

is a continuous positive sublinear functional on $X$. The polar of $K$ is the subset $K^{0}$ of $X^{*}$ defined by

$$
K^{0} \equiv\left\{f \in X^{*}: f(x) \leq 1 \text { for all } x \in K\right\}
$$

and is weak* compact convex and $0 \in$ int $K^{0}$. The gauge $p^{*}$ of $K^{0}$ on $X^{*}$ is continuous and weak* lower semi-continuous. We denote by $K^{00}$ the polar of $K^{0}$ in $X^{* *}$ and by $p^{* *}$ the gauge of $K^{00}$ on $X^{* *}$, and note that $K^{00}=\overline{\hat{K}}^{\omega *}$ and $\left.p^{* *}\right|_{\hat{X}}=p$, [7, Lemma 3.1(i), p. 255].

We now characterize Hausdorff weak upper semi-continuity by a density property. Such a characterization for subdifferentials of norms was given in [6, Theorem 3.1, p. 103] and was proved more generally for subdifferentials of proper lower semicontinuous convex functions in [1, Theorem 3.1, p. 98]. Since our theorem in Section 3 concerns gauges of bounded closed convex sets we include a direct proof for subdifferentials of gauges. 
THEOREM 2.1. Consider a Banach space $X$ and a bounded closed convex subset $K$ with $0 \in$ int $K$. The continuous gauge $p$ of $K$ has subdifferential mapping $x \mapsto \partial p(x)$ Hausdorff weak upper semi-continuous at $x \in X$ if and only if

$$
\widehat{\partial p(x)} \text { is weak }{ }^{*} \text { dense in } \partial p^{* *}(\hat{x}) \text {. }
$$

PROOF. Suppose that the density property does not hold. Then there exists $\mathfrak{F}_{0} \in$ $\partial p^{* *}(\hat{x})$ which we can strongly separate from $\overline{\partial \hat{\partial p(x)}}^{\omega *}$ by a weak* continuous linear functional $F_{0} \in S\left(X^{* *}\right)$. So there exists a weak* neighbourhood $N_{F_{0}}^{*}$ of 0 in $X^{* * *}$ generated by $F_{0}$ such that

$$
\mathfrak{F}_{0} \notin \widehat{\partial p(x)}+N_{F_{0}}^{*} .
$$

Since $\hat{K}^{0}$ is weak* dense in $K^{000}$, for each $n \in \mathbb{N}$ there exists $f_{n} \in K^{0}$ such that

$$
\left|\left(f_{n}-\mathfrak{F}_{0}\right)(\hat{x})\right|<\frac{1}{n} \quad \text { and } \quad\left|\left(f_{n}-\mathfrak{F}_{0}\right)\left(F_{0}\right)\right|<\frac{1}{n} .
$$

Since $\mathfrak{F}_{0} \in \partial p^{* *}(\hat{x})$ then $\mathfrak{F}_{0}(\hat{x})=p^{* *}(\hat{x})$ and $\mathfrak{F}_{0}(F) \leq p^{* *}(F)$ for all $F \in X^{* *}$. Then $\left|f_{n}(\hat{x})-p^{* *}(\hat{x})\right|<1 / n$ and, since $f_{n} \in K^{0}, f_{n}(y) \leq p(y)$ for all $y \in X$. So $f_{n}(\hat{x}) \geq$ $p^{* *}(\hat{x})-1 / n=p(x)-1 / n$ and $f_{n}(y-x) \leq p(y)-p(x)+1 / n$ for all $y \in X$. By the Brøndsted-Rockafellar theorem for each $n \in \mathbb{N}$ there exist $y_{n} \in X$ and $f_{y_{n}} \in$ $\partial p\left(y_{n}\right)$ such that

$$
\left\|f_{n}-f_{y_{n}}\right\| \leq \frac{1}{\sqrt{n}} \quad \text { and } \quad\left\|x-y_{n}\right\| \leq \frac{1}{\sqrt{n}}
$$

by [13, Theorem 3.17, p. 48]. But if the subdifferential mapping $x \mapsto \partial p(x)$ is Hausdorff weak upper semi-continuous at $x$, then, for sufficiently large $n \in \mathbb{N}$,

$$
f_{y_{n}} \in \partial p(x)+N_{F_{0}}
$$

where $N_{F_{0}}$ is the weak neighbourhood of 0 in $X^{*}$, the restriction of $N_{F_{0}}^{*}$ in $X^{* * *}$. However,

$$
\left|\left(f_{y_{n}}-\mathfrak{F}_{0}\right)\left(F_{0}\right)\right| \leq\left\|f_{y_{n}}-f_{n}\right\|+\left|\left(f_{n}-\mathfrak{F}_{0}\right)\left(F_{0}\right)\right| \leq \frac{1}{\sqrt{n}}+\frac{1}{n},
$$

which contradicts our original separation.

Conversely, suppose that

$$
\widehat{\partial p(x)} \text { is weak* dense in } \partial p^{* *}(\hat{x}) \text {. }
$$

Consider a weak neighbourhood $V$ of 0 in $X^{*}$. Now $V$ is the restriction of a weak ${ }^{*}$ neighbourhood $V^{*}$ of 0 in $X^{* * *}$. Since the subdifferential mapping $F \mapsto \partial p^{* *}(F)$ is Hausdorff weak* upper semi-continuous at $\hat{x} \in X^{* *}$, there exists $\delta>0$ such that

$$
\partial p^{* *}(B(\hat{x}, \delta)) \subseteq \partial p^{* *}(\hat{x})+\frac{1}{2} V^{*} \quad \text { but } \quad \partial p^{* *}(\hat{x}) \subseteq \widehat{\partial p(x)}+\frac{1}{2} V^{*}
$$

so $\partial p(B(x, \delta)) \subseteq \partial p(x)+V$.

It is instructive to see how the density property has implications for the density of extreme points. 
Corollary 2.2. A continuous gauge $p$ on a Banach space $X$ with subdifferential mapping $x \mapsto \partial p(x)$ Hausdorff weak upper semi-continuous at $x \in X$ satisfies:

(i) $\partial p^{* *}(\hat{x})=\overline{\operatorname{cosext}} \widehat{\partial p(x)}^{\omega *}$;

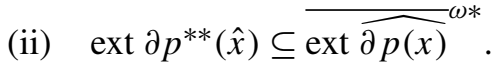

PROOF. Consider $\mathfrak{F}_{0}$ an extreme point of $\partial p^{* *}(\hat{x})$. Choquet's theorem [4, p. 77], gives us that weak* slices of $\partial p^{* *}(\hat{x})$, sets of the form

$$
\operatorname{Sl}\left(\partial p^{* *}(\hat{x}), F, \delta\right) \equiv\left\{\mathfrak{F} \in \partial p^{* *}(\hat{x}): \mathfrak{F}(F)>\sup \partial p^{* *}(\hat{x})(F)-\delta\right\},
$$

containing $\mathfrak{F}_{0}$ form a weak* neighbourhood base for $\mathfrak{F}_{0}$. It then follows from Theorem 2.1 that there exists an element of $\widehat{\partial p(x)}$ in the slice and, moreover, an extreme point of $\widehat{\partial p(x)}$. Since by the Krein-Milman theorem we have that $\partial p^{* *}(\hat{x})=$ ${\overline{\operatorname{co~ext~} \partial p^{* *}(\hat{x})}}^{\omega *}$, we deduce that

$$
\partial p^{* *}(\hat{x})=\overline{\operatorname{cosedt}}_{\partial \widehat{\partial p(x)}^{\omega *}} .
$$

But further, since $\widehat{\partial p(x)} \subseteq \partial p^{* *}(\hat{x})$, it follows that

$$
\operatorname{ext} \partial p^{* *}(\hat{x}) \subseteq \overline{\operatorname{ext} \widehat{\partial p(x)}}^{\omega *}
$$

by [4, Theorem 3.41, p. 78]. This concludes the proof.

\section{The continuity characterization}

A Banach space $X$ is an Asplund space if and only if its dual $x^{*}$ has the RadonNikodým property [13, Theorem 5.7, p. 82]. We exploit the following characterization of the Radon-Nikodým property to establish our theorem.

Proposition 3.1 [2, Corollary 3.76, (1) $\Leftrightarrow$ (3), p. 67]. A Banach space X has the Radon-Nikodým property if and only if every bounded closed convex subset $K$ of $X$ contains an extreme point of $\overline{\hat{K}}^{\omega^{*}}$.

THEOREM 3.2. A Banach space $X$ is an Asplund space if every continuous gauge $p$ on $X$ has a point $x_{0} \in X$ where the subdifferential mapping $x \mapsto \partial p(x)$ is Hausdorff weak upper semi-continuous and the subdifferential $\partial p\left(x_{0}\right)$ is weakly compact.

ProOf. Consider $K$ a bounded closed convex subset of $X^{*}$. We may assume that $0 \in K$. The support function $p$ of $K$ on $X$ is

$$
p(x)=\sup \{f(x): f \in K\} .
$$

Since $K$ is bounded, $p$ is continuous. Further, $p$ is the gauge of the set

$$
\{x \in X: p(x) \leq 1\}=K_{0} \equiv\{x \in X: f(x) \leq 1 \text { for all } f \in K\}
$$


which is a bounded closed convex subset of $X$ and $0 \in$ int $K_{0}$. Now the polar of $K_{0}$ in $X^{*}$,

$$
K_{0}^{0} \equiv\left\{f \in X^{*}: f(x) \leq 1 \text { for all } x \in K_{0}\right\},
$$

is a bounded weak* closed convex subset of $X^{*}$ and $K_{0}^{0}=\bar{K}^{\omega^{*}}$, by $[4$, Theorem 4.32, p. 119]. Consider $p^{*}$ the gauge of $K_{0}^{0}$ on $X^{*}$ and the polar of $K_{0}^{0}$ in $X^{* *}$,

$$
K_{0}^{00} \equiv\left\{F \in X^{* *}: F(f) \leq 1 \text { for all } f \in K_{0}{ }^{0}\right\}
$$

Finally, consider $p^{* *}$ the gauge of $K_{0}^{00}=K^{0}$ on $X^{* *}$. If the subdifferential mapping $x \mapsto \partial p(x)$ is Hausdorff weak upper semi-continuous at $x_{0} \in X$, then, by Theorem 2.1,

$$
\partial p^{* *}\left(\hat{x_{0}}\right)={\overline{\widehat{\partial p\left(x_{0}\right)}}}^{\omega^{*}}
$$

But if also $\partial p\left(x_{0}\right)$ is weakly compact in $X^{*}$ then

$$
\partial p^{* *}\left(\hat{x_{0}}\right)=\widehat{\partial p\left(x_{0}\right)} \subseteq \hat{X}^{*} .
$$

Now by the Krein-Milman theorem, $\partial p^{* *}\left(\hat{x_{0}}\right)$ has an extreme point, some $\hat{f}_{0} \in$ $\widehat{\partial p\left(x_{0}\right)}$. However, $\partial p^{* *}\left(\hat{x_{0}}\right)$ is an extreme subset of $K_{0}^{000}=K^{00}=\overline{\hat{K}}^{\omega^{*}}$, so $\hat{f}_{0}$ is an extreme point of $\overline{\hat{K}}^{\omega^{*}}$. Suppose that $f_{0} \notin K$. Then we can separate $f_{0}$ and $K$ by a weakly closed hyperplane. Then we can separate $\hat{f}_{0}$ and $\overline{\hat{K}}^{\omega^{*}}$ by a weak* closed hyperplane. But this contradicts $\hat{f}_{0} \in \overline{\hat{K}}^{\omega^{*}}$. So then $\overline{\hat{K}}^{\omega^{*}}$ has an extreme point in $K$. By Proposition 3.1 we have that $X^{*}$ has the Radon-Nikodým property and it follows that $X$ is an Asplund space.

\section{Remarks}

Our Theorem 3.2 goes some way towards an extension of the result of Godefroy et al. [12, Theorem 1, p. 494]. However, it is apparent that the weakly compact condition on the subdifferential, although satisfying the requirements of Proposition 3.1, is more stringent than is necessary. So, any advance with our line of argument requires us to explore further the relations given in Corollary 2.2.

\section{References}

[1] J. Benítez and V. Montesinos, 'Restricted weak upper semicontinuous differentials of convex functions', Bull. Aust. Math. Soc. 63 (2001), 93-100.

[2] R. D. Bourgin, Geometric Aspects of Convex Sets with the Radon-Nikodým Property, Springer Lecture Notes in Mathematics, 33 (Springer, New York, 1983).

[3] M. D. Contreras and R. Payá, 'On upper semicontinuity of duality mappings', Proc. Amer. Math. Soc. 121 (1994), 451-459.

[4] M. Fabian, P. Habala, P. Hájek, V. M. Santalucía, J. Pelant and V. Zizler, Functional Analysis and Infinite-Dimensional Geometry, CMS Books in Mathematics (Springer, New York, 2001). 
[5] C. Franchetti and R. Payá, 'Banach spaces with strongly subdifferentiable norm', Boll. Unione Mat. Ital. 7 (1993), 45-70.

[6] J. R. Giles, D. A. Gregory and B. Sims, 'Geometrical implications of upper semi-continuity of the duality mappings on Banach space', Pacific J. Math. 79 (1978), 99-109.

[7] J. R. Giles and W. B. Moors, 'A continuity property related to Kuratowski's index of noncompactness, its relevance to the drop property, and its implications for differentiabilty theory', J. Math. Anal. Appl. 178 (1993), 247-268.

[8] J. R. Giles and W. B. Moors, 'Generic continuity of restricted weak upper semi-continuous setvalued mappings', Set-Valued Anal. 4 (1996), 25-39.

[9] G. Godefroy, 'Some applications of Simons' inequality', Serdica. Math. J. 26 (2000), 59-78.

[10] G. Godefroy and V. Indumathi, 'Norm-to-weak upper semi-continuity of the duality and preduality mappings', Set-Valued Anal. 10 (2002), 317-330.

[11] G. Godefroy, V. Indumathi and F. Lust-Piquard, 'Strong subdifferentiability of convex functionals and proximinality', J. Approx. Theory 116 (2002), 397-415.

[12] G. Godefroy, V. Montesinos and V. Zizler, 'Strong subdifferentiability of norms and geometry of Banach spaces', Comment Math. Uni. Carolin. 36 (1995), 493-502.

[13] R. R. Phelps, Convex Functions, Monotone Operators and Differentiability, 2nd edn, Lecture Notes in Mathematics, 1364 (Springer, New York, 1993).

J. R. GILES, School of Mathematical and Physical Sciences, The University of Newcastle, New South Wales 2308, Australia e-mail: John.Giles@newcastle.edu.au 